\title{
Investigation of therapeutic effect of Saccharomyces boulardii and translocation in immunosuppressed rats infected with Shigella sonnei
}

Mustafa Behcet $^{1}$ - Ayse Demet Kaya ${ }^{2}$

${ }^{1}$ Department of Medical Microbiology, Bolu Abant Izzet Baysal University, School of Medicine, Bolu, Turkey

${ }^{2}$ Department of Medical Microbiology, Okan University, School of Medicine, Istanbul, Turkey

\section{ABSTRACT}

\begin{abstract}
Aim: To investigate the therapeutic effects of Saccharomyces boulardii (S. boulardii) and detect blood and tissue penetrations of $S$. boulardii and Shigella sonnei (S. sonnei) in immunosuppressed rats infected with $S$. sonnei.
\end{abstract}

Methods: Forty rats were divided into four groups: Group A (immunosuppressed, not-treated); Group B (immunosuppressed, treated with $S$. boulardii); Group C (immunosuppressed, infected with $S$. sonnei, treated with $S$. boulardii); Group D (immunosuppressed, infected with $S$. sonnei). After taking samples for blood cultures, the rats were sacrificed. The large bowel, liver, spleen and mesenteric lymph nodes (MLN) were removed for microbiological examination.

Results: $S$. boulardii in group B and $S$. sonnei in group D were isolated from blood in some rats. Statistical analysis of our data, showed that the numbers of translocated colonies in the liver and spleen were relatively higher for $S$. boulardii in Group B and for $S$. sonnei in Group D, without reaching levels of statistical significance. For MLN, colony counts in Group B was higher than Group $\mathrm{C}$ and A showing statistical significance.

Conclusion: The administration of $S$. boulardii showed promising results for the therapy of $S$. sonnei infection in immunosuppressed rats, but therapeutic usage of $S$. boulardii should be carefully assessed by taking into consideration the risk it poses versus potential benefits in risk groups.

Keywords: Saccharomyces boulardii, Shigella sonnei, immunosuppression, rat, translocation.

(C) 2020 experimentalbiomedicalresearch.com

$\triangle$ Dr. Mustafa Behçet

Depart. of Medical Microbiology, Bolu Abant Izzet Baysal University, School of Medicine, Bolu, Turkey

E mail: mustafabehcet@ibu.edu.tr

Received: 2020-02-19 / Revisions: 2020-03-03

Accepted: 2020-03-09 / Publication Date: 2020-06-13

\section{Introduction}

Intestinal infections due to Shigella spp. are worldwide endemic but it mainly occurs in developing countries. Four species of Shigella
(S); S. dysenteriae, S. flexneri, S. boydii and S. sonnei are the causative agents of shigellosis. Nearly two-thirds of the infections are caused by $S$. flexneri in low and middle-income countries.

S. sonnei is the leading species in high-income countries and the second most common species in low and middle-income countries. Immunodeficiencies lead to more severe clinical manifestations of Shigella infection 
including persistent or recurrent intestinal disease and bacteriemia [1]. Antimicrobial agents used as effective options in the treatment of shigellosis became limited due to global drug resistance [2].

Saccharomyces boulardii (S. boulardii) is a non-pathogenic yeast used in many countries in the treatment of non-specific diarrhea and in cases of gut flora impairment. Several mechanisms such as fungal antagonism, diminution of the pathogenic effects of bacterial toxins, stimulation of intestinal immune defenses and increased intestinal disaccharidase activity can possibly explain the actions of $S$. boulardii in diarrhea. The activity of $S$. boulardii has been extensively studied in the contexts of gastroenteritis and antibioticassociated diarrhea. The good tolerability of this yeast was shown and no serious adverse reactions have been reported despite a very slight potential risk of blood penetration of $S$. boulardii in immunodeficient individuals [3].

This study was conducted with the aim of investigating the therapeutic effects of $S$. boulardii in immunosuppressed rats infected with $S$. sonnei and detecting the presence of translocations of $S$. boulardii and $S$. sonnei in vulnerable hosts.

\section{Materials and methods}

These experiments were performed with the approval of the Ethics Committee of Duzce University School of Medicine (Decision no: 100-019). The animals which were used in the study were provided by Duzce University. The procedures were conducted according to routine animal care guidelines, and all experimental procedures complied with the Guide for the Care and Use of Laboratory Animals (1996).

In this study, forty male Wistar albino rats weighing $200 \pm 20 \mathrm{~g}$ were divided into four groups of 10 animals each: Group A (immunosuppressed, not treated), Group B (immunosuppressed, treated with $S$. boulardii), Group C (immunosuppressed, infected with $S$. sonnei, treated with $S$. boulardii) and Group D (immunosuppressed, infected with $S$. sonnei).

All rats were housed individually in stainless steel cages in an animal room at $20^{\circ} \mathrm{C}$ with a 12-hour light-dark cycle. All the rats were fed with a laboratory pellet diet and were allowed to have free access to water during the course of the experiments. Rats were decontaminated from antibiotics for 4 days by adding $2 \mathrm{mg} / \mathrm{ml}$ of streptomycin sulphate (Sigma, St. Louis, MO, USA) and 1500 units/ml penicillin-G (Sigma) to their water, which was prepared daily. Reduction of the gastrointestinal flora was confirmed by microscopic examination of Gram stained smears of fecal pellets and with aerobic cultures of feces for Gram-negative enteric bacteria.

The animals in all groups were given cyclophosphamide (Baxter Oncology, Germany) intraperitoneally at a dose of $200 \mathrm{mg} / \mathrm{kg}$. Group A was the immunosuppressed rats with no infection. On the fourth day after immunosuppression, Groups C and D were inoculated with $0.1 \mathrm{ml}$ of S. sonnei containing $9 \times 10^{8}$ viable cells by gavage route after having cultures of the bacteria in Brain Heart Infusion broth (HiMedia Laboratories, India) for 12 hours [4]. On the third day after the inoculation, $S$. sonnei was isolated from the fecal samples of all rats.

Lyophilized $S$. boulardii (Ultra-Levure; BIOCODEX Laboratories, Montrouge, France) was given to each rat at a single dose of $10 \mathrm{mg} /$ day by gavage route for Groups $\mathrm{B}$ and $\mathrm{C}$. Group A was given $0.1 \mathrm{ml}$ (same amount as other groups) of phosphate buffer saline (PBS), as this group was neither inoculated by $S$. boulardii nor by $S$. sonnei. $S$. boulardii 
administration was continued for 10 days for group B and for five days for group C. The rats were sacrificed under ether inhalation on day 10.

Prior to being sacrificed, blood samples $(2 \mathrm{ml})$ of rats were collected from inferior vena cava and inoculated into the bottles of BACTEC system (Becton Dickinson, Ireland).

After the rats were sacrificed; their large bowels, liver, spleen and MLN were removed for microbiologic examination. To evaluate the translocation by microbiological methods; the weights of the liver, spleen and nodes were recorded.

To assess $S$. boulardii and/or $S$. sonnei quantitatively, tissue pieces were minced with a scalpel, diluted by tenfold in $0.9 \% \mathrm{NaCl}$ and homogenized with a handled tissue tearer. (Ultra-Turrax T25, BioSpec Products, Bartlesville, OK, USA).

Diluted organ homogenates were transferred on Sabouraud dextrose agar (SDA) (HiMedia Laboratories, India) and Hektoen Enteric (HE) (HiMedia Laboratories, India) agar plates. The duration of the cultures were as follows: 7 days for blood cultures, 72 hours for SDA and 48 hours for $\mathrm{HE}$ agars at $35^{\circ} \mathrm{C}$. Microorganisms were identified by conventional methods and API 32E (BioMerieux, France) and API CAUX (BioMerieux, France) systems. Then, the number of colonies per tissue gram (cfu/g) was determined.

The translocation index shows the number of microorganisms per gram of tissue and was calculated by the following formula [5]:

(Cfu count x dilution coefficient x $10 \times 2$ ) / Tissue weight.

\section{Statistical Analysis}

The data was expressed as means \pm SD. Kruskal-Wallis and Mann-Whitney $U$ tests were used to compare the numbers of colonies between the groups. The differences were considered as being significant at $p<0.05$.

\section{Results}

The number of colonies of the microorganisms isolated from the cultures of MLN, liver and spleen specimens and the results of blood cultures were determined for each group.

In Group A, in immunosuppressed rats, blood and tissue cultures were negative and no translocations were seen.

In Group B (immunosuppressed, treated-with$S$. boulardii), the results showed that $S$. boulardii translocated and it systemically spread to extraintestinal sites of liver, spleen and MLN in some rats. Of 10 rats, one yielded positive results in the liver, spleen, MLN and blood; one yielded positive results in spleen and MLN; one yielded positive results in MLN and blood; and, lastly, four rats yielded positive results in only the MLN cultures. The isolated organism was $S$. boulardii from the MLN, liver and spleen specimens. $S$. boulardii was also isolated from the blood specimens of two rats in which translocations were observed.

In Group C (immunosuppressed, infected-withS.sonnei, treated-with- $S$. boulardii), neither $S$. boulardii nor $S$. sonnei was isolated from the cultures of MLN, liver, spleen and/or blood specimens.

In Group D (immunosuppressed, infected-withS. sonnei), the results of the cultures were as follows: one rat yielded positive results in the liver, spleen, MLN and blood specimens; one yielded positive results in the liver, MLN and blood specimens; and one rat yielded positive results in MLN for $S$. sonnei.

The numbers of translocated colonies are listed in Table 1 and are expressed as cfu. The numbers of translocated colonies in liver and spleen were relatively higher for $S$. boulardii in Group B and higher for S. sonnei in Group D, 
but the difference was not statistically significant $(p>0.05)$. For MLN, colony counts in Group B was higher than in Groups C and A, which was statistically significant $(p=0.005)$. In Group D, colonies of $S$. sonnei was high, but not with statistical significance $(p=0.068)$. certain agents, including Giardia intestinalis, Rotavirus, Entamoeba histolytica, Helicobacter pylori, ETEC, Cryptosporidium parvum as well as in the treatment of diarrhea associated with HIV [9-11]. S. boulardii has been found to show a protective effect in Clostridium difficile

Table 1. Numbers of colonies in the liver, spleen, MLN and blood in the four experimental.

\begin{tabular}{|c|c|c|c|c|}
\hline Parameters & $A(n=10)$ & $B(n=10)$ & $C(n=10)$ & $D(n=10)$ \\
\hline Liver* (cfu/g/10 $\left.{ }^{5}\right)$ & 0.00 & $363.60 \pm 1149.80$ & 0.00 & 194. $20 \pm 409.41$ \\
\hline Spleen **(cfu/g/10 $)$ & 0.00 & $1039.10 \pm 2360.01$ & 0.00 & $210.50 \pm 666.65$ \\
\hline MLN***(cfu/g/10 $)$ & 0.00 & $47273.70 \pm 75043.94$ & 0.00 & $10378.70 \pm 17400.4$ \\
\hline Bacteriemia/fungemia & 0 & 2 & 0 & 2 \\
\hline
\end{tabular}

$\boldsymbol{n}=$ number of rats. $\boldsymbol{B}:$ For $S$. boulardii

$* * *(p=0.001)$ (Kruscal-Wallis test); $(p=0.005)$ groups $A-B, B-C$ (Mann-Whitney U test)

D: For S. sonnei

$* * *(p<0.05)($ Kruscal-Wallis test $):(p=0.068)$ groups $A-D, C-D($ Mann-Whitney $U$ test $)$

\section{Discussion}

$S$. boulardii is widely used for clinical conditions and predominantly for the prevention of diarrhea [6,7]. The effects of $S$. boulardii are related to its inhibitory effects on the growth of intestinal microorganisms and to the neutralization of toxins, however the mechanisms underlying such effects could not be fully identified. Furthermore, S. boulardii has been shown to increase the activity of brush border disaccharidases in human volunteers and in patients with congenital sucrase isomaltase deficiency. In addition, an enhanced release of secretory IgA into the intestinal lumen supports the immune system [8].

Many studies have been conducted to investigate the activity of $S$. boulardii against associated colitis [12,13]. Studies showed a protective effect against Salmonella typhimurium and Shigella flexneri in the intestinal tracts of conventional or gnotobiotic mice [14]. The effects of orogastric administration of $S$. boulardii on the jejunal villi was studied by Dias et al. [15] in rats infected with Vibrio cholerae and their data showed the inhibition of the action of the cholera toxin on enterocytes by $S$. boulardii. Sheele et al. [16] showed the efficiency of $S$. boulardii in patients in whom the duration and severity of cholera was reduced. Zbinden et al. [17] investigated the influence of $S$. boulardii on Salmonella typhimurium and Yersinia enterocolitica under in vitro conditions and 
their results showed that $S$. boulardii inhibited either the growth of both bacteria or their invasion into HeLa cells, so they suggested to study these effects in vivo as well.

In recent years, invasive fungal infections have been frequently reported worldwide in parallel to with the increase in risky population such as patients with chronic or debilitating diseases, who receive immunosuppressive drugs broad spectrum antibiotics, and parenteral nutrition and who were administered central venous catheter [18].

Microbial translocation is defined as the passage of viable microbes from the gastrointestinal (GI) tract to extraintestinal sites, such as the MLN, spleen, liver, kidneys, and blood [19]. Overall, $S$. boulardii is considered to be a safe and well tolerated agent, but recently, numerous studies reported fungemia after $S$. boulardii treatment for risk groups [17,20-28]. Some studies emphasize the importance of blood penetration after $S$. boulardii usage in immunosuppressed patients resulting in $S$. boulardii associated fungemia $[29,30]$. For this reason, the therapeutic usage of probiotics should be carefully evaluated by taking into consideration its risks as well as its potential benefits.

In a study investigating the ability of orally administered viable $S$. boulardii in inhibiting translocation of Candida albicans from the gastrointestinal tract in antibioticdecontaminated, specific pathogen-free mice, orally administered $S$. boulardii was shown to decrease the incidence of Candida albicans translocation to the MLN, liver and kidneys [19].

Peret Filho et al. [31] studied the translocation and histological alterations in the terminal ileum, liver and spleens of immunosuppressed mice under $S$. boulardii treatment. The results of this study showed that $S$. boulardii administration decreased the bacterial translocation to the liver and spleen in a dose dependant manner. Low $S$. boulardii translocation to MLN was observed in some animals. In our study, in Group B, translocation to MLN was significantly higher $(p=0.005)$. The translocation to spleen and liver was also high, but the difference was not statistically significant $(p>0.05)$. In evaluation of the blood cultures, two rats (20\%) in Group B developed fungemia due to Saccharomyces cerevisiae representing the translocation of $S$. boulardii. Comparing our results with Peret et al. [31] our findings also support the relative protection with $S$. boulardii in immunosuppressed rats. However, reported cases in clinical trials and development of fungemia in two immunosuppressed rats showed the importance of probable fungemia during treatment.

Shigellosis is currently an important public health problem. Shigella bacteriemia is rare but associated with a high mortality rate. Immunosuppressed patients, malnourished children, and elderly people are at risk of serious complications and bacteriemia $[2,32]$. In experiments on white mice, $S$. sonnei strains were shown to be capable of penetrating into the blood for a short period of time [33]. Over the past decades, Shigella strains have progressively become resistant to most of the widely used antimicrobials [2]. For this reason, alternative therapeutic approaches are under investigation. In our study, we investigated the therapeutic effects of $S$. boulardii in S. sonnei infection, which is the predominant cause of Shigellosis in our country and the translocation rate of $S$. sonnei and $S$. boulardii in immunosuppressed hosts. The results of our study showed that in some rats in the D group $S$. sonnei cause bacteremia and caused translocation in the MLN, liver and spleen. These results showed the importance of 
bacterial translocation in immunosuppressed rats. In group $C$, in which the immunosuppressed rats were infected with $S$. sonnei and treated with $S$. boulardii, no translocation and no growth in blood cultures was observed.

\section{Conclusion}

Both S. boulardii and S. sonnei caused translocation resulting in fungemia/ bacteriemia in immunosuppressed rats when they were applied individually; but $S$. boulardii administration to $S$. sonnei infected rats seemed to result in the inhibition of translocation and bacteriemia. $S$. boulardii administration is found to be effective in the treatment of $S$. sonnei infections in immunosuppressed rats under in vivo conditions, but potential risk of developing fungemia in risk groups must always be considered during the therapeutic use of $S$. boulardii.

\section{Acknowledgements}

This study was produced from the doctoral thesis.

Funding: There is no financial support and sponsorship

Conflict of Interest: The authors declare that they have no conflict of interest.

Ethical statement: This experimental study was reviewed and approved by the local ethics committee (Decision no; 100-019).

\section{ORCID iD of the author(s)}

Mustafa Behcet / 0000-0002-5676-6983

Ayse Demet Kaya / 0000-0001-8224-8242

\section{References}

[1]Kotloff KL, Riddle MS, Platts-Mills JA, et al. Shigellosis. Lancet. 2018; 391(10122):801-12.
[2]Peirano G, Souza FS, Rodrigues DP. Shigella Study Group. Frequency of serovars and antimicrobial resistance in Shigella spp. from Brazil. Mem Inst Oswald Cruz. 2006; 101(3):245-50.

[3]Saint-Marc T, Blehaut H, Musial C, et al. AIDS-related diarrhea: a double-blind trial of Saccharomyces boulardii. Sem Hop Paris. 1995; 71(23):735-41.

[4]Rene K, Vidal PKE, Christine FM, et al. Shigella dysenteriae type-1 induced diarrhea in rats. Jpn J Infect Dis. 2005; 58(6):335-37.

[5]Güngör S, Kurultay N, Şener AG, et al. Demonstration of bacterial translocation in an experimental obstructive jaundice model in rats. Klimik Derg. 2003; 16(3):121-25.

[6]Sharif MR, Kashani HH, Ardakani AT, et al. The effect of a yeast probiotic on acute diarrhea in children. Probiotics Antimicrob Proteins. 2016; 8(4):211-14.

[7]Guarino A, Guandalini S, Lo Vecchio A. Probiotics for prevention and treatment of diarrhea. J Clin Gastroenterol. 2015; 49(1):37-45.

[8]Plein K, Hotz J. Therapeutic effects of Saccharomyces boulardii on mild residual symptoms in a stable phase of Crohn's disease with special respect to chronic diarrhea a pilot study. Z Gastroenterol. 1993; 31(2):129-34.

[9]Castaneda Guillot C, Garcia Bacallao E, Santa Cruz Dominguez M, et al. Effects of Saccharomyces boulardii in children with chronic diarrhea, especially cases of giardiasis. Rev Mex Puericultura Pediatria 1995;2:12-16.

[10]Feng JR, Wang F, Qiu X, et al. Efficacy and safety of probiotic-supplemented triple therapy for eradication of Helicobacter pylori in children: a systematic review and network meta-analysis. Eur J Clin Pharmacol. 2017; 73(10):1199-208. 
[11]Czerucka D, Rampal P. Experimental effects of Saccharomyces boulardii on diarrheal Pathogens. Microbes Infect. 2002; 4(7):73339.

[12] Valdés-Varela L, Gueimonde M, RuasMadiedo P. Probiotics for prevention and treatment of Clostridium difficile infection. In: Mastrantonio P, Rupnik M. (eds) Updates on Clostridium difficile in Europe. Advances in Experimental Medicine and Biology, Springer, Cham 2018.vol 1050. p. 161-76.

[13]Allen SJ. The potential of probiotics to prevent Clostridium difficile infection. Infect Dis Clin North Am. 2015; 29(1):13544.

[14] Rodrigues ACP, Nardi RM, Bambirra EA, et al. Effect of Saccharomyces boulardii against experimental oral infection with Salmonella typhimurium and Shigella flexeneri in conventional and gnotobiotic mice. J Appl Bacteriol. 1996; 81(3):251-56.

[15]Dias RS, Bambirra EA, Silva ME, et al. Protective effect of Saccharomyces boulardii against the cholera toxin in rats. Braz J Med Biol Res. 1995; 28(3):323-25.

[16] Sheele J, Cartowski J, Dart A, et al. Saccharomyces boulardii and bismuth subsalicylate as low-cost interventions to reduce the duration and severity of cholera. Pathog Glob Health. 2015; 109(6):275-82.

[17]Zbinden R, Gönczi EE, Altwegg M. Inhibition of Saccharomyces boulardii (nom. inval.) on cell invasion of Salmonella typhimurium and Yersinia enterocolitica. Microb Ecol Health Dis. 1999; 11(3):15862.

[18] Cassone M, Sera P, Mondello F, et al. Outbreak of Saccharomyces cerevisiae subtype boulardii fungemia in patients neighboring those treated with a probiotic preparation of the organism. J Clin Microbiol. 2003; 41(11):5340-43.
[19]Berg R, Bernasconi P, Fowler D, et al. Inhibition of Candida albicans translocation from the gastrointestinal tract of mice by oral administration of Saccharomyces boulardii. J Infect Dis. 1993; 168(5):1314-18.

[20]Burkhardt O, Kohnlein T, Pletz M, et al. Saccharomyces boulardii induced sepsis: successful therapy with voriconazole after treatment failure with fluconazole. Scand J Infect Dis. 2005; 37(1):69-72.

[21]Lestin F, Pertschy A, Rimek D. Fungemia after oral treatment with Saccharomyces boulardii in a patient with multiple comorbidities. Dtsch Med Wochenschr. 2003; 128(48):2531-33.

[22] Riquelme AJ, Calvo MA, Guzman AM, et al. Saccharomyces cerevisiae fungemia after Saccharomyces boulardii treatment in immunocompromised patients. J Clin Gastroenterol. 2003; 36(1):41-43.

[23] Lherm T, Monet C, Nougiere B, et al. Seven cases of fungemia with Saccharomyces boulardii in critically ill patients. Intensive. Care Med. 2002; 28(6):797-801.

[24] Romanio MR, Coraine LA, Maielo VP, et al. Saccharomyces cerevisiae fungemia in a pediatric patient after treatment with probiotics. Rev Paul Pediatr. 2017; 35(3):361-64.

[25] Kara I, Yıldırım F, Özgen Ö, et al. Saccharomyces cerevisiae fungemia after probiotic treatment in an intensive care unit patient. J Mycol Med. 2018; 28(1):218-21.

[26] Roy U, Jessani LG, Rudramurthy SM, et al. Seven cases of Saccharomyces fungaemia related to use of probiotics. Mycoses. 2017; 60(6):375-80.

[27]Lolis N, Veldekis D, Moraitou H, et al. Saccharomyces boulardii fungaemia in an intensive care unit patient treated with caspofungin. Crit Care. 2008; 12(2):414. 
[28] Appel-da-Silva MC, Narvaez GA, Perez LRR, et al. Saccharomyces cerevisiae var. boulardii fungemia following probiotic treatment. Med Mycol Case Rep. 2017; 18:15-17.

[29] Martin IW, Tonner R, Trivedi J, et al. Saccharomyces boulardii probioticassociated fungemia: questioning the safety of this preventive probiotic's use. Diagn Microbiol Infect Dis. 2017; 87(3):286-88.

[30]Eren Z, Gurol Y, Sonmezoglu M, et al. Saccharomyces cerevisiae fungemia in an elderly patient following probiotic treatment. Mikrobiyol Bul. 2014; 48(2):35155.

[31]Peret Filho LA, Pena FJ, Bambirra EA, et al. Dose effect of oral Saccharomyces boulardii treatments on morbidity and mortality in immunosuppressed mice. J Med Microbiol. 1998; 47(2):111-16.

[32] Morduchowicz G, Huminer D, SiegmanIgra Y, et al. Shigella bacteremia in adults. A report of five cases and review of the literature. Arch Intern Med. 1987; 147(11):2034-37.

[33] Suptel EA, Maksimovich NA. Experimental study of bacteremia and its role in the pathogenesis of dysentery. Arkh Patol. 1977; 39(2):17-22. 